\title{
Albumin-Bilirubin (ALBI) and Monocyte to Lymphocyte Ratio (MLR)-Based Nomogram Model to Predict Tumor Recurrence of AFP-Negative Hepatocellular Carcinoma
}

\author{
Shuqi Mao' \\ Xi Yu' \\ Yuying Shan' \\ Rui Fan ${ }^{2}$ \\ Shengdong $\mathrm{Wu}^{\prime}$ \\ Caide Lu' \\ 'Department of Hepatopancreatobiliary \\ Surgery, Ningbo Medical Center Lihuili \\ Hospital, Ningbo University, Ningbo, \\ Zhejiang, 315040, People's Republic of \\ China; ${ }^{2}$ Medical Quality Management \\ Office, Ningbo Medical Center Lihuili \\ Hospital, Ningbo University, Ningbo, \\ Zhejiang, 315040, People's Republic of \\ China
}

Correspondence: Caide Lu; Shengdong Wu

Department of Hepatopancreatobiliary Surgery, Ningbo Medical Center Lihuili Hospital, Ningbo University, Ningbo,

Zhejiang, 3 I5040, People's Republic of China

Tel +86 I3957800900; +86 I3567886669

Email lucaide@nbu.edu.cn;

13567886669@I39.com
Purpose: In this study, we aimed to develop a novel liver function and inflammatory markers-based nomogram to predict recurrence-free survival (RFS) for AFP-negative $(<20$ $\mathrm{ng} / \mathrm{mL}$ ) HCC patients after curative resection.

Patients and Methods: A total of 166 pathologically confirmed AFP-negative HCC patients were included at the Ningbo Medical Center Lihuili Hospital. A LASSO regression analysis was used for data dimensionality reduction and element selection. Univariate and multivariate Cox regression analyses were performed to identify the independent risk factors relevant to RFS. Finally, clinical nomogram prediction model for RFS of HCC was established. Nomogram performance was assessed via internal validation and calibration curve statistics. Receiver operating characteristic (ROC) and decision curve analysis (DCA) curve were used to validate the performance and clinical utility of the nomogram.

Results: Multivariate Cox regression analysis indicated that ALBI grade (hazard ratio, [HR] $=2.624,95 \%$ confidence interval $[\mathrm{CI}]: 1.391-4.949, P=0.003)$, INR $(\mathrm{HR}=2.605,95 \% \mathrm{CI}$ : $1.061-6.396, P=0.037)$, MLR $(\mathrm{HR}=1.769,95 \% \mathrm{CI}: 1.073-2.915, P=0.025)$ and MVI (HR $=4.726$, 95\% CI: 2.365-9.444, $P<0.001)$ were independent prognostic factors of RFS. Nomogram with independent factors was established and achieved a better concordance index of 0.753 (95\% CI: $0.672-0.834)$ for predicting RFS. The ROC found that the area under curve (AUC) was consistent with the $\mathrm{C}$-index and the sensitivity was $85.4 \%$. The risk score calculated by nomogram could divide AFP-negative HCC patients into high-, moderate- and low-risk groups $(P<0.05)$. DCA analysis revealed that the nomogram could augment net benefits and exhibited a wider range of threshold probabilities by the risk stratification than the AJCC T and BCLC stage in the prediction of AFP-negative HCC recurrence.

Conclusion: The ALBI grade- and MLR-based nomogram prognostic model for RFS showed high predictive accuracy in AFP-negative HCC patients after surgical resection.

Keywords: HCC, MLR, ALBI grade, AFP-negative, recurrence-free survival, nomogram

\section{Introduction}

Hepatocellular carcinoma (HCC) is the sixth most commonly diagnosed cancer and the third leading cause of cancer-related death in the world according to 2020 global cancer statistics. ${ }^{1}$ Potentially curative therapy recommended for HCC patients with early-stage tumors consists of surgical resection, liver transplantation or local ablation in clinical practice. For the vast majority of HCC patients, surgical 
resection is the mainstay of curative treatment options. In addition, surgical resection provides better clinical outcomes than local ablation particularly among patients with well-preserved hepatic function. ${ }^{2}$ However, recurrence and metastasis are the main drivers of poor prognosis for operable HCC patients, ${ }^{3}$ which is the main cause of death in the long-term follow-up assessment.

Since the identification of serum alpha-fetoprotein (AFP) in the 1970s, it has been a well-established diagnostic and prognostic indicator of increased tumor virulence and is associated with worse tumor phenotype and aggressiveness in HCC. ${ }^{4-6}$ However, a retrospective analysis found that approximately $31 \%$ of radiographically apparent HCC patients do not present with elevated serum AFP levels, ${ }^{7}$ which largely limits prognosis prediction and disease diagnosis. Some studies even suggested that the postoperative detection of serum AFP was useless and should be abandoned because of the poor sensitivity in AFP-negative HCC patients. ${ }^{8,9}$ Elevated serum AFP may also be observed in benign conditions commonly encountered in clinical practice, such as liver inflammation and cirrhosis. In the present study, we wanted to explore whether liver inflammatory biomarkers and functional detection could be regarded as the prognostic prediction factors for AFP-negative $\mathrm{HCC}$ recurrence when the value of AFP for the diagnosis and detection of HCC recurrence remains controversial. Liver function is another independent prognosticator to predict tumor recurrence used in operative models. ${ }^{10,11}$ Johnson PJ et al proposed that the ALBI grade was refinement of the Child-Pugh grade and has been widely validated and evidenced. ${ }^{12}$

In addition, chronic inflammation induces changes especially in the hepatic immune system, damages hepatic epithelial cells, and allows tumor cells to easily evade immune surveillance. ${ }^{13}$

A handful of inflammatory markers easily calculated by preoperative complete blood count and liver function tests, such as MLR, PLR, NLR, PNI, SIRI, and APRI et al, have been reported to be associated with HCC patient survival post curative surgical resection. ${ }^{14-16}$

Nomograms have been considered a reliable tool to integrate and quantify significant risk factors for disease prognosis. ${ }^{17,18}$ An accurate prognosis estimation can help surgeons choose appropriate therapeutic measures for special patients based on a risk-benefit assessment. However, how to accurately predict and find a uniform scheme or standard is a common problem faced by clinicians. Therefore, we aimed to develop a nomogram model to predict the probability of recurrence-free survival (RFS) in AFP-negative HCC patients after surgical resection. The prognostic prediction accuracy will be evaluated by calibration curves, and patients will be divided into different risk groups according to the risk score.

\section{Patients and Methods}

\section{Patients}

A total of 166 AFP-negative HCC patients who underwent hepatic resection at Ningbo Medical Center Lihuili Hospital from September 2015 to January 2021 were included. The inclusion criteria were as follows: (1) pathologically diagnosed HCC; (2) Child-Pugh A or B classification; (3) treated by intended cure resection, which was defined as negative margins with no residual tumor based on the histological examination; and (4) preoperative AFP negative $(<20 \mathrm{ng} / \mathrm{mL})$. The exclusion criteria were as follows: (1) received preoperative anticancer medication; (2) history of other cancers; and (3) incomplete clinical or follow-up data. The study was approved by the ethics committee of Ningbo Medical Center Lihuili Hospital (Approval number: KY2021PJ218). All research procedures were in compliance with the relevant guidelines and regulations. Informed consent was obtained from all patients prior to inclusion. We confirmed that this study was conducted in accordance with the Declaration of Helsinki.

\section{Laboratory Examination and Follow-Up}

Laboratory examinations included hepatitis B and C immunology, alanine aminotransferase (ALT), alkaline phosphatase (ALP), $\gamma$-glutamyl transpeptidase (GGT), international normalized ratio (INR), aspartate aminotransferase (AST), prothrombin time (PT), albumin (ALB), total bilirubin (TB) and direct bilirubin (DB); complete blood count included white blood cell count, neutrophil count, lymphocyte count, monocyte count, and platelet count. The albuminbilirubin (ALBI) score was computed by the formula, -0.085 $\times($ albumin $\mathrm{g} / \mathrm{l})+0.66 \times \log ($ bilirubin $\mu \mathrm{mol} / \mathrm{l}), \mathrm{HCC}$ patients were stratified into 3 groups according to previously described cut-offs resulting in 3 grades: ALBI grade 1 $(\leq-2.60)$, grade $2(>-2.60$ to -1.39$)$ and grade 3 $(>-1.39){ }^{12}$ The inflammatory markers were calculated by the following formula: GLR $=\gamma$-glutamyl transpeptidase/ lymphocyte, SII $=$ platelet $\times$ neutrophil/lymphocyte, ANRI $=$ aspartate aminotransferase $(\mathrm{U} / \mathrm{L}) /$ neutrophil, SIRI $=$ monocyte $\times$ neutrophil/lymphocyte, MLR $=$ monocyte/ 
lymphocyte, $\mathrm{PNI}=$ serum albumin $(\mathrm{g} / \mathrm{L})+5 \times$ lymphocyte, APRI $=$ aspartate aminotransferase $(\mathrm{U} / \mathrm{L}) /$ platelet, NLR = neutrophil/lymphocyte, PLR = platelet/lymphocyte, ALRI $=$ aspartate aminotransferase $(\mathrm{U} / \mathrm{L}) /$ lymphocyte.

The cut-off value of the laboratory index was set according to the Health Industry Standard of the People's Republic of China published by the National Health Commission of the People's Republic of China. Patients were defined as hypertensive based on the "gold standard", and had at least three consecutive measurements of systolic blood pressure (SBP) $>140 \mathrm{~mm} \mathrm{Hg}$ and/or diastolic blood pressure (DBP) $>90 \mathrm{~mm} \mathrm{Hg}$. Controls had SBP and DBP of $<120 \mathrm{~mm} \mathrm{Hg}$ and $<80 \mathrm{~mm} \mathrm{Hg}$, respectively, and first-degree relatives had no family history of hypertension. Type 2 diabetes mellitus (T2DM) was diagnosed according to the World Health Organization criteria: (1) fasting glucose level $>7 \mathrm{mmol} / \mathrm{l}$; (2) 2-hour oral glucose tolerance test showing a glucose level of $\geq 11.1 \mathrm{mmol} / \mathrm{l}$; (3) haemoglobin A1c $\geq 6.5 \%$, or (4) clinical diagnosis of the disease. Anatomic or nonanatomic resection was performed after the clinical evaluation, and all the obtained surgical specimens were histologically assessed by different pathologists. Pathological examination results included pathological differentiation, microvascular invasion (MVI), tumor diameter, number of tumors, cirrhosis, tumor capsule, and portal vein tumor thrombosis (PVTT).

Baseline data were collected from our clinical database. AFP-negative HCC patients were consistently followed-up after curative resection at intervals of 3 months. Follow-up was aimed at determination of RFS. RFS was calculated from the date of curative resection to the date when tumor recurrence was diagnosed. The preoperative and tumor recurrence diagnoses were based on criteria set forth in the guidelines for the diagnosis and treatment of primary liver cancer in China. ${ }^{19}$

\section{Statistical Analysis}

Quantitative variables are reported as medians and interquartile ranges (age and tumor diameter are reported as mean and standard deviation), while categorical variables are presented as absolute counts and percentages. Survival curves were calculated using the Kaplan-Meier method and the Log rank test. LASSO regression analysis was used for data dimensionality reduction and element selection. Independent prognostic factors of tumor recurrence was identified by univariate and multivariate Cox proportional hazards regression. Subsequently, a nomogram was formulated to predict RFS based on the results of multivariate Cox regression analysis. Nomogram performance was assessed via internal validation and calibration curve statistics (concordance index was calculated to measure discrimination with 1000 bootstrapping techniques). A receiver operating characteristic (ROC) curve was used to validate the nomogram model performance. Each patient had a total risk score (NomoScore: nomogram risk score) for risk stratification of RFS according to the nomogram model. Patients were divided into different risk groups (low-; moderate-; high-) with the cut-off points automatically calculated using X-tile software ${ }^{20}$ (version 3.6.1; Yale University, New Haven, CT, USA). Decision curve analysis (DCA) was conducted to determine the clinical benefit of the nomogram by quantifying the net benefits along with the increase in threshold probabilities.

Univariate, multivariate Cox regression analyses and ROC curve were performed using SPSS 25.0 (IBM Corporation, 2020, USA). LASSO regression, nomogram, DCA, and survival figures were performed or plotted using $\mathrm{R}$ version 3.6.2 (http://www.r-project.org/), with package dependencies: "rms", "glamet", "ggDCA", "rmda" "survival", "survminer", and "ggpubr". $P<0.05$ was considered statistically significant.

\section{Results}

\section{Prognostic Outcomes of HCC}

A total of 166 AFP-negative HCC patients who met the inclusion criteria were enrolled from the 625 pathologically diagnosed patients. The median follow-up time was 20 months (1-61 months). The 1-, 2-, and 3-year recurrence-free rates were $85.7 \%, 74.0 \%$, and $69.0 \%$, respectively. All baseline characteristics are summarized in Tables 1 and 2 .

\section{Dimensionality Reduction and Element Selection}

The LASSO coefficient profiles of the features were plotted. The optimum parameter (lambda) selection in the LASSO model performed tenfold cross-validation through minimum criteria. The partial likelihood deviance (binomial deviance) curve is presented versus log (lambda). Dotted vertical lines are shown at the optimum values by performing lambda.min and lambda.1se. Finally, ALP, ALBI, INR, PNI, NLR, MLR, MVI, number of tumors, tumor diameter, tumor capsule, and PVTT were selected according to the optimum value corresponding to the minimum value of lambda (Figure 1). 
Table I Baseline and Clinicopathological Characteristics of AFPNegative HCC Patients

\begin{tabular}{|c|c|c|}
\hline Variables & $\begin{array}{c}\text { Recurrence-Free } \\
\text { Group }(\mathrm{N}=\mid 25)\end{array}$ & $\begin{array}{c}\text { Recurrence } \\
\text { Group }(\mathrm{N}=41)\end{array}$ \\
\hline \multicolumn{3}{|l|}{ Gender } \\
\hline Male & 106 (84.8\%) & 37 (90.2\%) \\
\hline Female & 19 (I5.2\%) & $4(9.8 \%)$ \\
\hline Age, years & $55.0 \pm 11.3$ & $61.0 \pm 9.5$ \\
\hline \multicolumn{3}{|l|}{ HBV infection } \\
\hline Positive & 99 (79.2\%) & 36 (87.9\%) \\
\hline Negative & $26(20.8 \%)$ & $5(12.2 \%)$ \\
\hline \multicolumn{3}{|l|}{ Anti-HBV } \\
\hline Yes & 47 (37.6\%) & 8 (19.5\%) \\
\hline No & 78 (62.4\%) & 33 (80.5\%) \\
\hline \multicolumn{3}{|l|}{ Hypertension } \\
\hline Positive & $36(28.8 \%)$ & 15 (36.6\%) \\
\hline Negative & 89 (7I.2\%) & 26 (63.4\%) \\
\hline \multicolumn{3}{|l|}{ Diabetes } \\
\hline Positive & 17 (I3.6\%) & $6(14.6 \%)$ \\
\hline Negative & 108 (86.4\%) & 35 (85.4\%) \\
\hline \multicolumn{3}{|l|}{ ALT, U/L } \\
\hline$\leq 50$ & 103 (82.4\%) & 32 (78.0\%) \\
\hline$>50$ & $22(17.6 \%)$ & $9(22.0 \%)$ \\
\hline \multicolumn{3}{|l|}{ ALP, U/L } \\
\hline$\leq 125$ & 107 (85.6\%) & 28 (768.3\%) \\
\hline$>125$ & 18 (I4.4\%) & $13(31.7 \%)$ \\
\hline \multicolumn{3}{|l|}{ GGT, U/L } \\
\hline$\leq 60$ & 73 (58.4\%) & 22 (53.7\%) \\
\hline$>60$ & 52 (4I.6\%) & 19 (46.3\%) \\
\hline \multicolumn{3}{|l|}{ PT, seconds } \\
\hline$\leq 13$ & 104 (83.2\%) & 34 (82.9\%) \\
\hline$>13$ & 21 (16.8\%) & 7 (I7.1\%) \\
\hline \multicolumn{3}{|l|}{$\mathrm{DB}, \mathrm{umol} / \mathrm{l}$} \\
\hline$\leq 8$ & 105 (84.0\%) & 32 (78.0\%) \\
\hline$>8$ & $20(16.0 \%)$ & $9(22.0 \%)$ \\
\hline \multicolumn{3}{|l|}{ INR } \\
\hline$\leq 1$ & 36 (28.8\%) & $6(14.6 \%)$ \\
\hline$>1$ & 89 (7I.2\%) & 35 (85.4\%) \\
\hline \multicolumn{3}{|l|}{ Pathological } \\
\hline Poor & 29 (23.2\%) & II (26.8\%) \\
\hline Moderate & 79 (63.2\%) & $28(68.3 \%)$ \\
\hline Well & 17 (I3.6\%) & $2(4.9 \%)$ \\
\hline \multicolumn{3}{|l|}{ MVI } \\
\hline Positive & 40 (32.0\%) & 28 (68.3\%) \\
\hline Negative & 85 (68.0\%) & $13(31.7 \%)$ \\
\hline
\end{tabular}

(Continued)
Table I (Continued)

\begin{tabular}{|l|c|c|}
\hline Variables & $\begin{array}{c}\text { Recurrence-Free } \\
\text { Group(N=125) }\end{array}$ & $\begin{array}{c}\text { Recurrence } \\
\text { Group(N=4I) }\end{array}$ \\
\hline $\begin{array}{c}\text { Cirrhosis } \\
\text { Positive } \\
\text { Negative }\end{array}$ & $58(46.4 \%)$ & $19(46.3 \%)$ \\
$22(53.6 \%)$ & $(53.7 \%)$ \\
\hline $\begin{array}{l}\text { Number of tumors } \\
\text { Solitary }\end{array}$ & $108(86.4 \%)$ & $29(70.7 \%)$ \\
Multiple & $17(13.6 \%)$ & $12(29.3 \%)$ \\
\hline Tumor diameter, cm & $4.31 \pm 2.27$ & $5.88 \pm 3.32$ \\
\hline Tumor capsule & $16(12.8 \%)$ & $11(26.8 \%)$ \\
Positive & $109(87.2 \%)$ & $30(73.2 \%)$ \\
\hline Negative & $4(3.2 \%)$ & $6(14.6 \%)$ \\
\hline PVTT & $121(96.8 \%)$ & $35(85.4 \%)$ \\
\hline Positive & Negative &
\end{tabular}

Abbreviations: $\mathrm{HBV}$, hepatitis B Virus; ALT, alanine aminotransferase; ALP, alkaline phosphatase; GGT, $\gamma$-glutamyl transpeptidase; PT, prothrombin time; DB, direct bilirubin; INR, international normalized ratio; MVI, microvascular invasion; PVTT, portal vein tumor thrombosis.

\section{Development and Validation of Nomogram Prediction Model}

We incorporated the ALP, ALBI, INR, PNI, NLR, MLR, MVI, number of tumors, tumor diameter, tumor capsule, and PVTT into the univariate and multivariate Cox regression equations. Multivariate Cox regression analysis indicated that ALBI (hazard ratio, $[\mathrm{HR}]=2.624,95 \%$ confidence interval [CI]: 1.391-4.949, $P=0.003)$, INR $(\mathrm{HR}=2.605,95 \% \mathrm{CI}: 1.061-6.396, P=0.037)$, MLR (HR $=1.769,95 \%$ CI: $1.073-2.915, P=0.025)$ and MVI $(\mathrm{HR}=4.726,95 \%$ CI: 2.365-9.444, $P<0.001)$ were independent prognostic factors of RFS (Table 3). Incorporating ALBI, INR, MVI, and MLR, the nomogram (Figure 2A) achieved a better concordance index of 0.753 (95\% CI: $0.672-0.834)$ with 1000 bootstrap samples to measure discrimination in predicting 1-, 2-, and 3-year RFS rates. The ROC curve found that the area under curve (AUC) was consistent with the C-index (0.753; 95\% CI: $0.672-0.834$ ) and the sensitivity was $85.4 \%$ (Figure 2B). The calibration curves for the 1-, 2- and 3 -year RFS rates were largely overlapped with the standard lines (Figure 2C-E). The resultant nomogram could accurately distinguish the RFS of AFP-negative HCC patients and had better consistency between the predicted probability and the observed probability of RFS. 
Table 2 ALBI Grade and Inflammatory Markers of AFP-Negative HCC Patients

\begin{tabular}{|c|c|c|}
\hline Variables & $\begin{array}{l}\text { Recurrence-Free Group } \\
(\mathrm{N}=\mid 25)\end{array}$ & $\begin{array}{l}\text { Recurrence Group } \\
(\mathrm{N}=41)\end{array}$ \\
\hline \multicolumn{3}{|l|}{ ALBI grade } \\
\hline I & $93(74.4 \%)$ & 21 (5I.2\%) \\
\hline 2 & $32(25.6 \%)$ & 20 (48.8\%) \\
\hline 3 & $0(0.0 \%)$ & $0(0.0 \%)$ \\
\hline GLR & $31.05(4.44,416.67)$ & $50.00(8.64,531.43)$ \\
\hline PNI & $50.8(26.5,73.3)$ & 46.I $(3 \mid .4,58.3)$ \\
\hline APRI & $0.17(0.04,2.16)$ & $0.22(0.06,1.01)$ \\
\hline ALRI & $16.67(6.3,360)$ & $27.00(6.07,342.5)$ \\
\hline ANRI & $8.80(1.75,63.53)$ & $11.61(1.51,51.25)$ \\
\hline SII & $296.18(65.63,3227.54)$ & $381.27(64.29,3502)$ \\
\hline NLR & $2.00(0.47,16.5)$ & $2.33(0.95,25.75)$ \\
\hline PLR & $103.75(42.5,256.15)$ & $106.15(30.91,340)$ \\
\hline MLR & $0.30(0.12,1.7)$ & $0.33(0.17,5.17)$ \\
\hline SIRI & $0.83(0.23,21.45)$ & I.0I $(0.27,12.55)$ \\
\hline \multicolumn{3}{|l|}{$\begin{array}{l}\text { Child-Pugh } \\
\text { grade }\end{array}$} \\
\hline A & $122(97.6 \%)$ & 40 (97.6\%) \\
\hline B & $3(2.4 \%)$ & I (2.4\%) \\
\hline \multicolumn{3}{|l|}{$\mathrm{AJCC}$} \\
\hline \multicolumn{3}{|l|}{ T stage } \\
\hline I-II & 106 (84.80\%) & 35 (85.4\%) \\
\hline III-IV & $19(12.0 \%)$ & $6(14.6 \%)$ \\
\hline \multicolumn{3}{|l|}{ BCLC stage } \\
\hline$A$ & $92(73.6 \%)$ & 34 (82.9\%) \\
\hline B & $18(14.4 \%)$ & $4(9.8 \%)$ \\
\hline C & $15(12.0 \%)$ & $3(7.3 \%)$ \\
\hline
\end{tabular}

Abbreviations: ALBI, albumin-bilirubin; GLR, $\gamma$-glutamyl transpeptidase to lymphocyte ratio; PNI, prognostic nutritional index; APRI, aspartate aminotransferase to platelet ratio; ALRI, aspartate aminotransferase to lymphocyte ratio; ANRI, aspartate aminotransferase to neutrophil ratio; SIl, systemic ImmuneInflammation; NLR, neutrophil to lymphocyte; PLR, platelet to lymphocyte ratio; MLR, monocyte to lymphocyte ratio; SIRI, systemic inflammation response index AJCC, American Joint Committee on Cancer; BCLC, Barcelona Clinic Liver Cancer.

\section{Prognostic Assessment and Risk Stratification}

AFP-negative HCC patients were divided into different risk groups based on the total risk scores calculated by the nomogram models to judge the discriminatory abilities of the nomogram for RFS. The optimal cut-off points were autocalculated by $\mathrm{X}$-tile software. The risk scores calculated can divide AFP-negative HCC patients into the high-risk group (>116), moderate risk group (54-116) and low-risk group $(<54)$. Survival curves were calculated to compare the RFS rates in three different risk groups using the Kaplan-Meier method, and the results showed a significant discriminatory ability for recurrence risks in the patients based on the nomogram risk scores $(P<0.05$, Figure 3A). DCA analysis revealed that the nomogram model could augment net benefits and exhibited a wider range of threshold probabilities by risk stratification than the American Joint Committee on Cancer (AJCC) T and Barcelona Clinic Liver Cancer (BCLC) stage in the prediction of AFP-negative HCC recurrence (Figure 3B).

\section{Discussion}

The nomogram prediction model that enabled risk assessment of recurrence after curative resection has been developed and validated in HCC patients with negative serum AFP. The constructed nomogram prediction model included ALBI, INR, MLR, and MVI which were screened through LASSO and multivariate Cox regression analysis. Individuals with higher total points had a greater probability of recurrence-free survival. For example, if ALBI was $>-2.6$, with INR $>1, \operatorname{MLR}=1$, and positive MVI of HCC patient, the corresponding total point was 80 , and the probability of 1-year, 2-year, and 3-year RFS was approximately $86 \%, 73 \%, 68 \%$. The developed nomogram could accurately distinguish the probability of recurrence and shows a better consistency between the predicted probability and actual frequency of RFS, with a C-index of 0.753 (95\% CI: $0.672-0.834$ ). The nomogram provided intuitive and easy-to-understand clinical tools to guide a rational and personalized subsequent therapeutic choice for clinical surgeons.

ALBI grade was calculated using only serum albumin and total bilirubin as a simple and statistical evaluation method for hepatic function. ${ }^{12}$ In a multicentre cohort, Chan et $\mathrm{al}^{21}$ indicated that the overall prognostic performance of ALBI grade-based and Child-Pugh-based BCLC was similar, because of the high concordance of a weighted $\kappa$ value. Hiraoka $\mathrm{A}$ et $\mathrm{al}^{22}$ demonstrated that the assessment ability of hepatic reserve function shown by ALBI grade was adequate for use in the Japan Society of Hepatology (JSH) guidelines instead of the ChildPugh classification (CP) and liver damage classification. Previous studies found that ALBI grade predicted overall survival (OS) more accurately than $\mathrm{CP}$ in patients with HCC who underwent curative hepatic resection. ${ }^{23,24}$ Chan et $\mathrm{al}^{11}$ also found that the ERASL model using ALBI grade and MVI provided better discriminatory power for the risk stratification of early recurrence. Several studies even constructed nomogram models based on ALBI grade and other clinical data for predicting the recurrence of HCC undergoing curative hepatic 


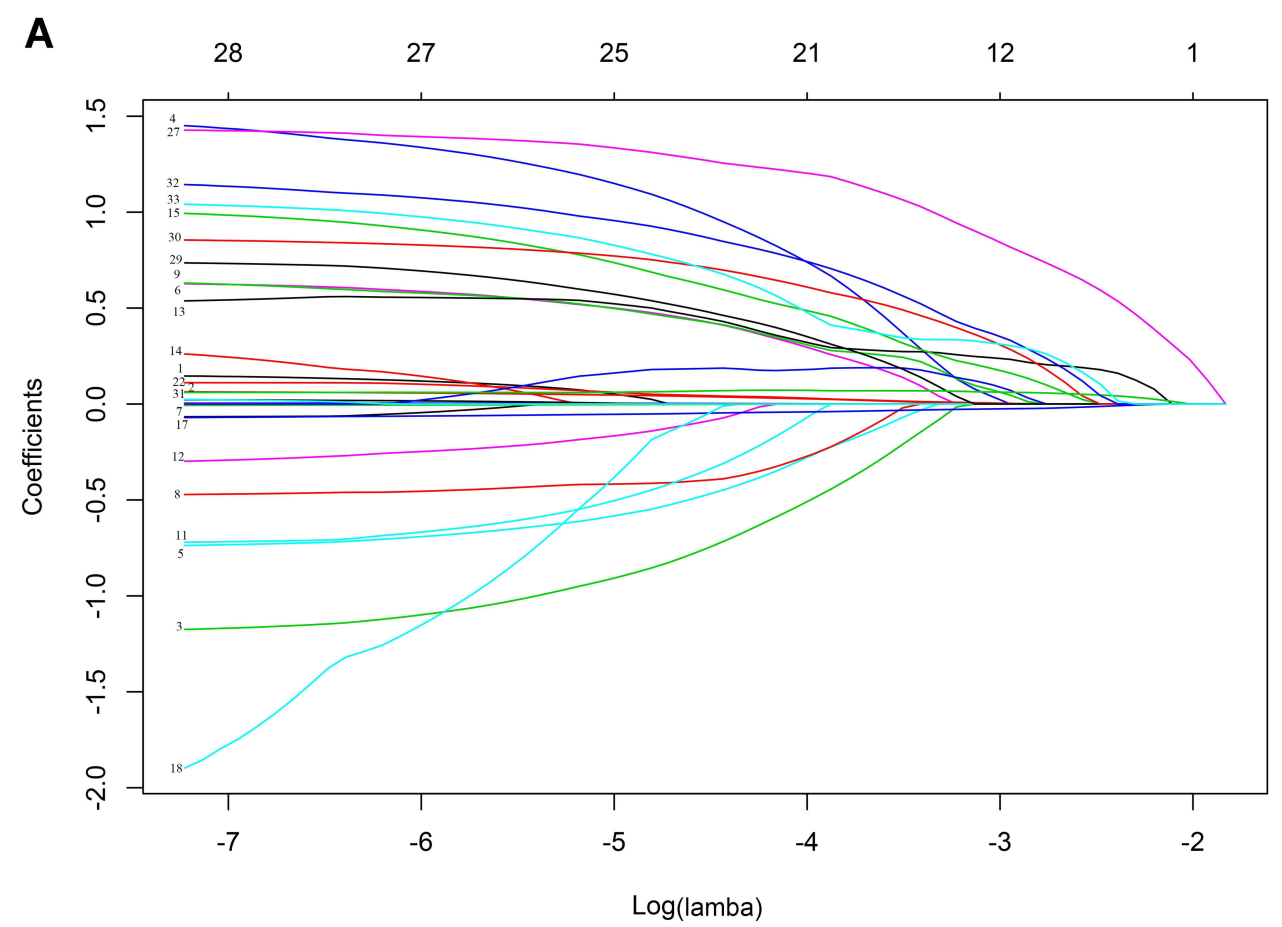

B

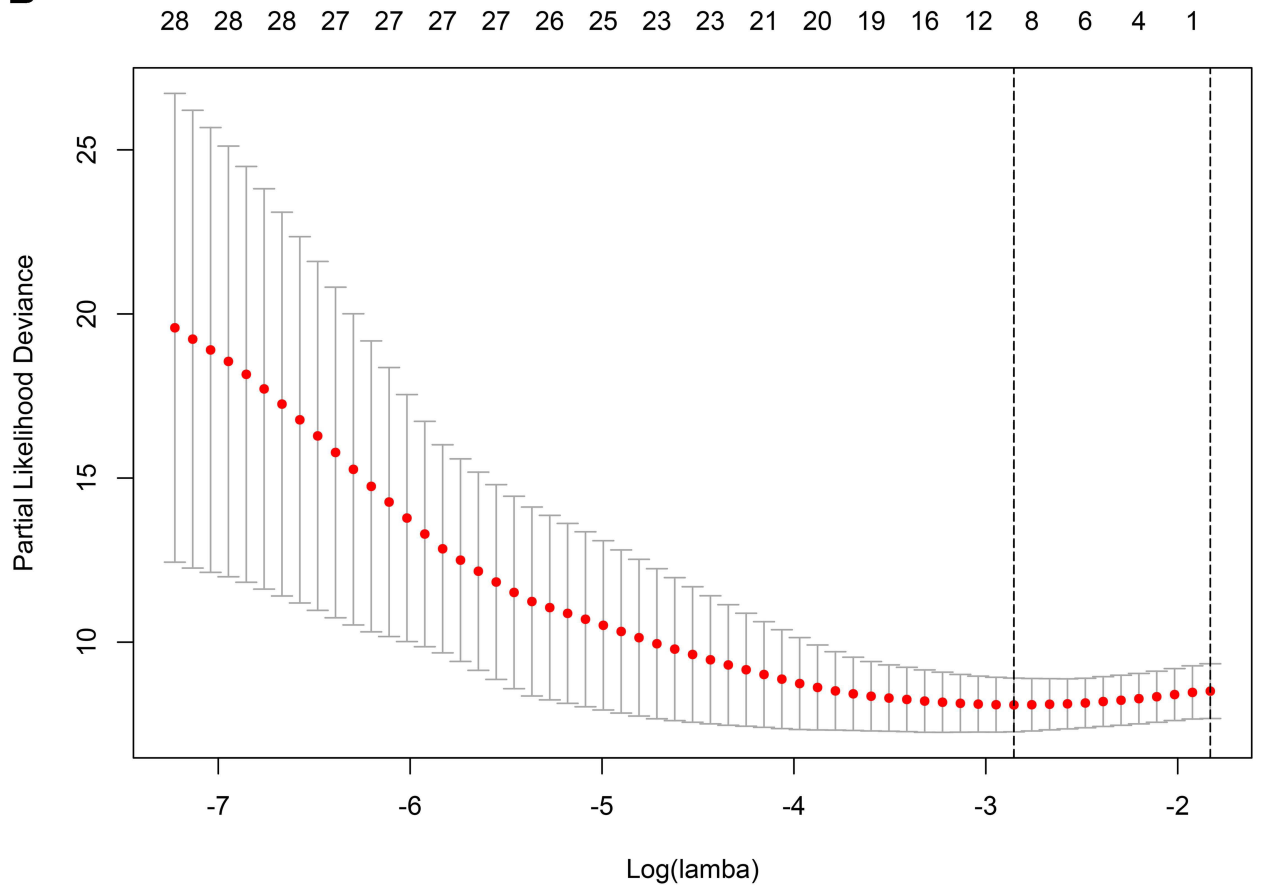

Figure I Nomogram model elements selection of RFS using the LASSO regression model. (A) The LASSO coefficient profiles of the clinical features. (B) Optimum parameter (lambda) selection in the LASSO model performed ten-fold cross-validation.

resection. ${ }^{24-26}$ In the present study, we first found that the ALBI grade was significant independent prognostic factor of AFP-negative HCC following curative resection. The developed ALBI-based nomogram could accurately predict the RFS of AFP-negative HCC patients and had better consistency between the predicted and observed probabilities of RFS.

As a prototypical inflammation-related cancer, approximately $90 \%$ of HCC burden associated with prolonged hepatitis due to viral hepatitis, excessive 
Table 3 Univariate and Multivariate Cox Analyses for RFS of AFP-Negative HCC Patients

\begin{tabular}{|l|c|c|c|c|}
\hline \multirow{2}{*}{ Variables } & \multicolumn{2}{|c|}{ Univariate Analysis } & \multicolumn{2}{c|}{ Multivariate Analysis } \\
\cline { 2 - 5 } & $\boldsymbol{P}$ & HR (95\% CI) & $\boldsymbol{P}$ & HR (95\% CI) \\
\hline ALP >I25 & 0.039 & $2.004(1.037-3.873)$ & & $2.624(1.391-4.949)$ \\
ALBI grade & 0.001 & $2.719(1.470-5.027)$ & 0.003 & $2.605(1.061-6.396)$ \\
INR >I & 0.032 & $2.614(1.088-6.28 I)$ & 0.037 & \\
PNI & 0.004 & $0.943(0.905-0.981)$ & & $1.769(1.073-2.915)$ \\
NLR & 0.012 & $1.090(1.019-1.167)$ & & $4.726(2.365-9.444)$ \\
MLR & 0.004 & $1.828(1.207-2.768)$ & $<.025$ & \\
MVI & $<0.001$ & $3.845(1.982-7.457)$ & & \\
Number of tumors & 0.026 & $2.146(1.094-4.208)$ & & \\
Tumor diameter & $<0.001$ & $1.210(1.097-1.334)$ & & \\
Tumor capsule & 0.010 & $2.514(1.248-5.064)$ & & \\
PVTT & 0.002 & $4.045(1.675-9.769)$ & & \\
\hline
\end{tabular}

Abbreviations: ALP, alkaline phosphatase; ALBI, albumin-bilirubin; INR, international normalized ratio; PNI, prognostic nutritional index; NLR, neutrophil to lymphocyte; MLR, monocyte to lymphocyte ratio; MVI, microvascular invasion; PVTT, portal vein tumor thrombosis.

alcohol intake, nonalcoholic fatty liver disease (NAFLD) or nonalcoholic steatohepatitis (NASH). ${ }^{27}$ The immune microenvironment and inflammatory markers are all parts of the systemic inflammatory response and play an important role in cancer development, progression, invasion, and metastasis. ${ }^{28,29}$ The present study first confirmed that the MLR could be used to determine the recurrence of AFP-negative HCC patients after curative resection. As a systemic inflammatory marker in the peripheral blood, the MLR is low in cost of examination and simple to calculate by monocytes and lymphocytes and can be measured repeatedly. Activated circulating monocytes can secrete multiple proinflammatory cytokines that are involved in tumor development and progression. ${ }^{30}$ In contrast, a low lymphocyte count is associated with systemic inflammatory responses and is known to promote cancer progression through effects on cell-mediated immunity. ${ }^{31}$ A meta-analysis demonstrated that low MLR was significantly correlated with increased OS in patients with $\mathrm{HCC}$, regardless of the sample size, type of publication, NOS score, and cut-off value of the MLR. ${ }^{32} \mathrm{Wu} \mathrm{Y}$ et $\mathrm{al}^{33}$ reported that the MLR combined with clinical risk factors is helpful for clinicians to identify high-risk HCC patients with early recurrence and improve the prognosis of HCC patients. Although we found a correlation between the MLR and RFS in AFP-negative HCC patients, but not the other inflammatory markers, the mechanism is not very precise.
Microvascular invasion (MVI) was defined as the presence of tumor cells within a vascular lumen lined by endothelium that is visible only by microscopy ${ }^{34,35}$ and considered as a critical determinant of early recurrence and survival of HCC. Tumor cells can spread and metastasize in the liver to form a portal vein tumor thrombus or multiple lesions or distant metastasis in the presence of MVI. ${ }^{36}$ Residual intrahepatic metastases have been frequently considered as the main cause of early recurrences within 2 years of tumor resection. Recent studies have indicated that high-risk MVI patients did significantly worse with regard to both recurrence and survival, ${ }^{6,37,38}$ and multiple features of MVI carry prognostic significance for HCC. In this study, our results indicated that MVI was also an important prognostic factor for AFP-negative HCC, which was consistent with a previous study. ${ }^{39}$ INR was proposed to standardize prothrombin time (PT) reporting by the World Health Organization in the early 1980s and was an excellent measure of liver synthetic function, as decreasing the production of factor VII occurs in the cirrhotic liver as disease severity worsens. ${ }^{40}$ The INR has also been recommended to evaluate the survival of patients with severe liver disease and has been included in the MELD score. Wang $\mathrm{Y}$ et $\mathrm{al}^{41}$ demonstrated that nomograms based on INR and other clinical indices exhibited high efficacy in predicting OS and RFS in HCC patients after hepatectomy. Haruki $\mathrm{K}$ et $\mathrm{al}^{42}$ found that a high preoperative prothrombin time-international normalized ratio to albumin ratio was an independent and significant risk factor for poor disease-free and overall survival after 


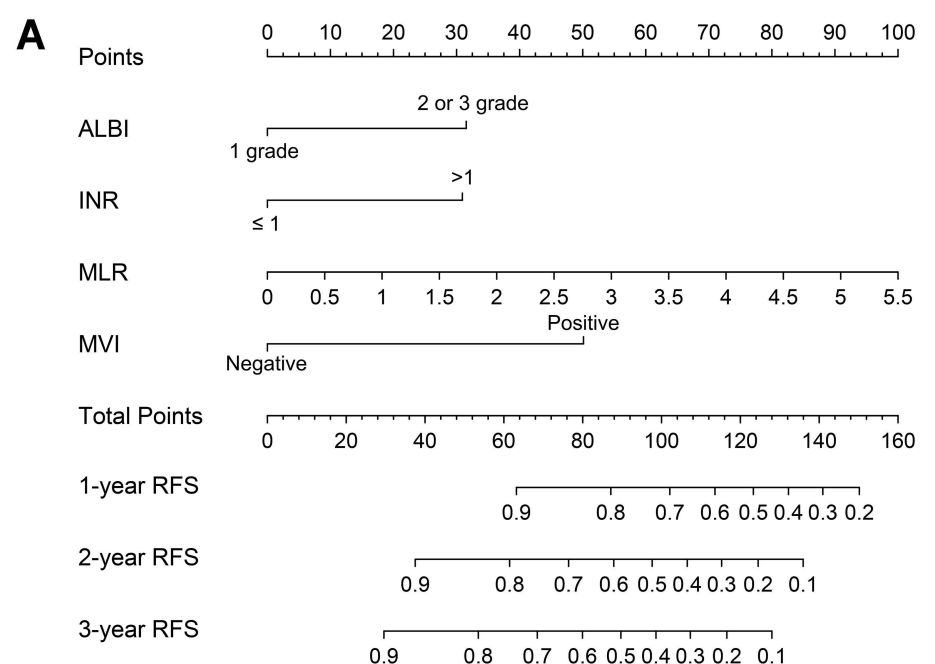

C

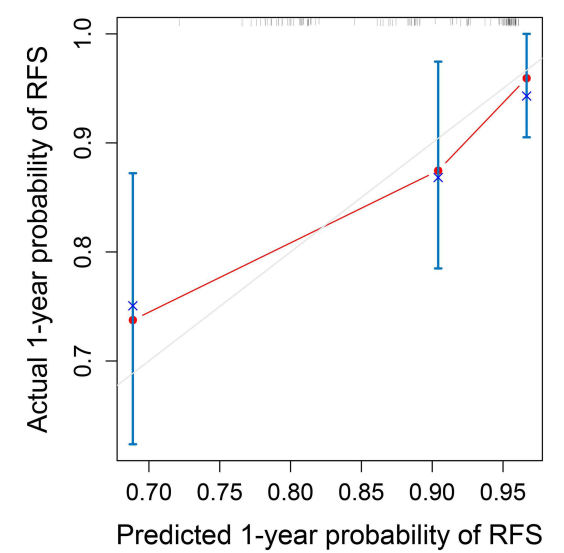

D

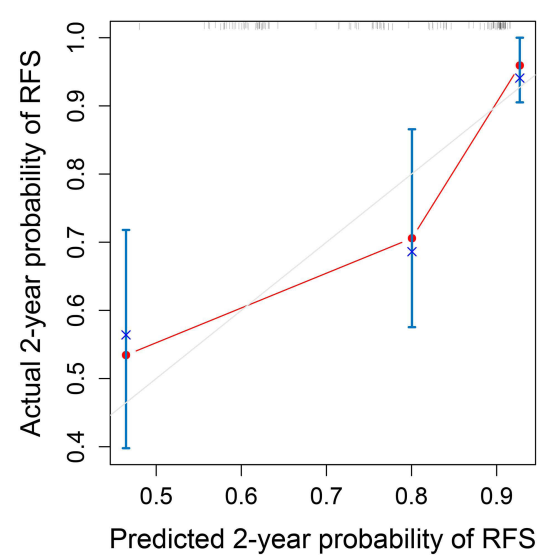

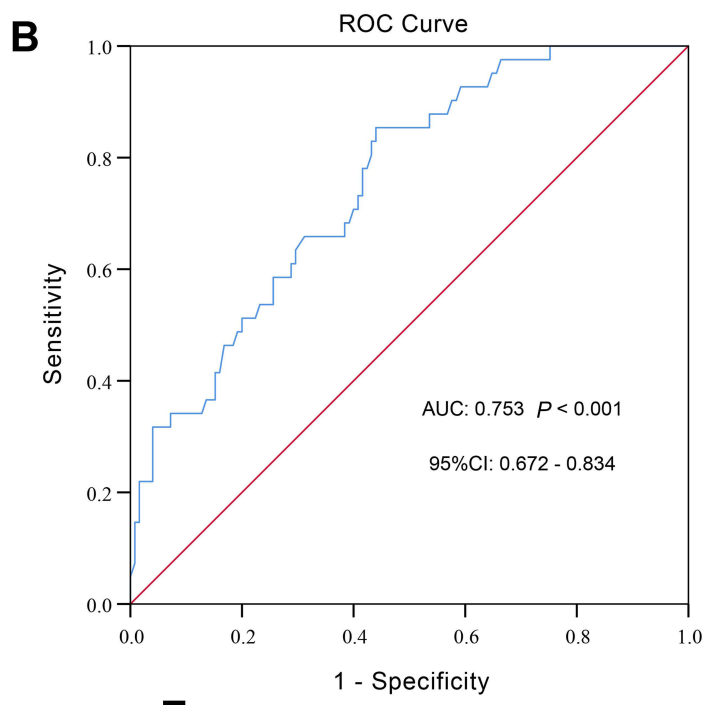

E

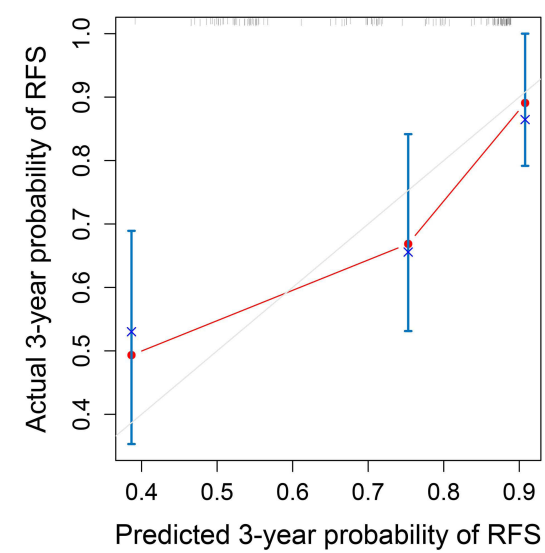

Figure 2 Developed prognosis nomogram model for RFS. (A) Nomogram model. (B) Receiver operating characteristic curve for the nomogram model. (C-E) Calibration curves plots of nomogram for predicting I-year, 2-year, and 3-year probability of recurrence-free survival.

Abbreviations: ALBI, albumin-bilirubin; INR, international normalized ratio; MLR, monocyte to lymphocyte ratio; MVI, microvascular invasion; RFS, recurrence-free survival. ROC, receiver operating characteristic; AUC, area under curve.

hepatic resection for HCC. In this study, the INR was also found to be an independent prognostic factor of AFPnegative $\mathrm{HCC}$ as an excellent measure of liver synthetic function.

A LASSO regression analysis model was used to construct data dimensionality reduction and element selection in our study. Overfitting, optimism, and miscalibration might be addressed and accounted for during model development by applying bootstrapping techniques and LASSO regression. ${ }^{43}$ In addition, DCA analysis revealed that the nomogram model could augment net benefits and exhibited a wider range of threshold probabilities by risk stratification in the prediction of AFP-negative HCC recurrence. Although the majority of nomogram studies commonly split the dataset into two subsets randomly, a development sample and a validation sample, this was not done in our study. However, we only developed the internal validation of the nomogram prediction model with a representative sample size to avoid statistical inefficiency or wasted data. ${ }^{44}$

There are limitations in this study. First, this research is a single center retrospective cohort study, and a prospective cohort is needed to further confirm the predictive value of our nomogram model in prognosis. Second, the nomogram model was only constructed based on a limited clinical database, and other prognosisrelated factors and biomarkers need to be identified and incorporated to further promote the accuracy of the AFPnegative HCC nomogram model. 
A

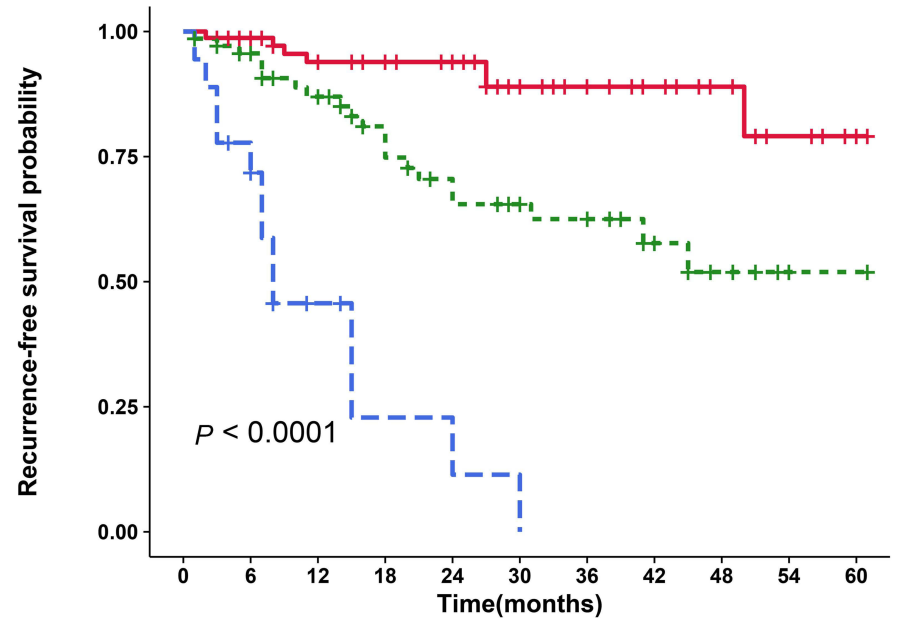

\section{Number at risk}

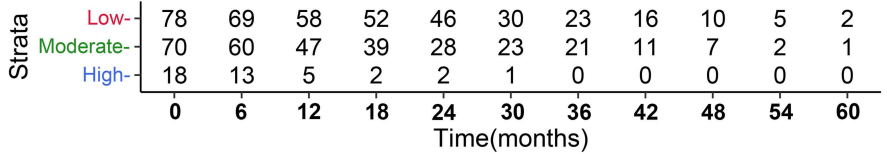

B

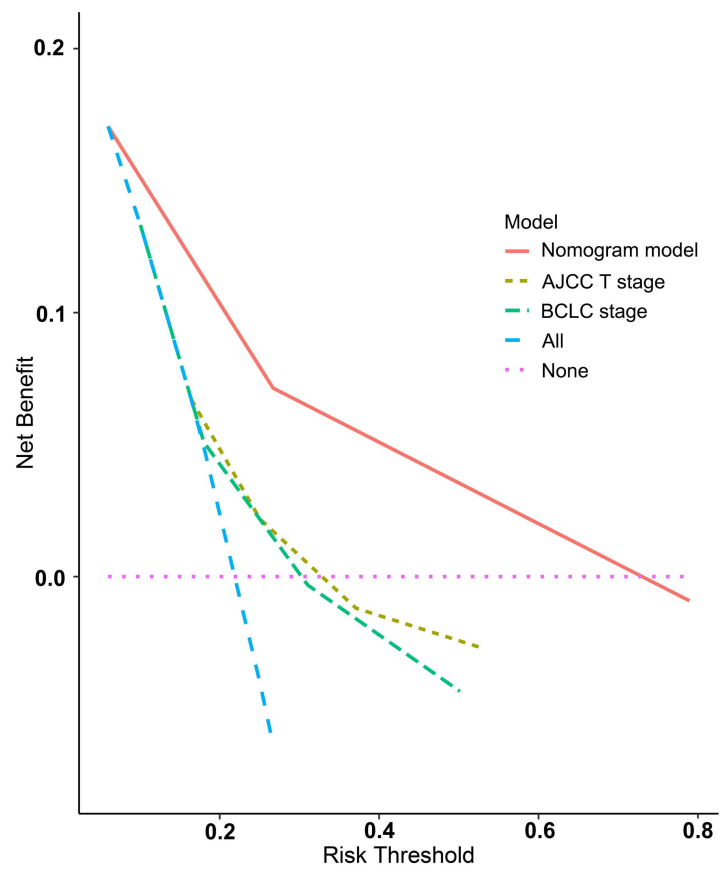

Figure 3 Prognostic assessment and risk stratification of developed nomogram model. (A) Risk stratification for recurrence-free survival. (B) Decision curve analysis. Abbreviations: AJCC, American Joint Committee on Cancer; BCLC, Barcelona Clinic Liver Cancer.

\section{Conclusion}

In conclusion, the ALBI grade- and MLR-based nomogram prognostic model for RFS exhibits great potential predictive accuracy in AFP-negative HCC patients after surgical resection.

\section{Funding}

Our research was supported by the grants from Ningbo Health Branding Subject Fund (PPXK2018-03), Science and Technology program of Zhejiang Health (2021KY1035). Medical Health Science and Technology Project of Zhejiang Province (2019ZD047).

\section{Disclosure}

The authors report no conflicts of interest in this work.

\section{References}

1. Sung H, Ferlay J, Siegel RL, et al. Global Cancer Statistics 2020: GLOBOCAN estimates of incidence and mortality worldwide for 36 cancers in 185 countries. CA Cancer J Clin. 2021;71(3):209-249.

2. Chan AC, Chan SC, Chok KS, et al. Treatment strategy for recurrent hepatocellular carcinoma: salvage transplantation, repeated resection, or radiofrequency ablation? Liver Transplantation. 2013;19(4):4 11-419. doi:10.1002/lt.23605

3. Erstad DJ, Tanabe KK. Prognostic and therapeutic implications of microvascular invasion in hepatocellular carcinoma. Ann Surg Oncol. 2019;26(5):1474-1493. doi:10.1245/s10434-019-07227-9
4. Mulé S, Galletto Pregliasco A, Tenenhaus A, et al. Multiphase liver MRI for identifying the macrotrabecular-massive subtype of hepatocellular carcinoma. Radiology. 2020;295(3):562-571. doi:10.1148/ radiol.2020192230

5. Song P, Tobe RG, Inagaki Y, et al. The management of hepatocellular carcinoma around the world: a comparison of guidelines from 2001 to 2011. Liver Int. 2012;32(7):1053-1063. doi:10.1111/j.1478-3231. 2012.02792.x

6. Mao S, Yu X, Yang Y, et al. Preoperative nomogram for microvascular invasion prediction based on clinical database in hepatocellular carcinoma. Sci Rep. 2021;11(1):13999. doi:10.1038/s41598-021-93528-7

7. Agopian VG, Harlander-Locke MP, Markovic D, et al. Evaluation of patients with hepatocellular carcinomas that do not produce $\alpha$ Fetoprotein. JAMA Surg. 2017;152(1):55-64. doi:10.1001/jamasurg. 2016.3310

8. Siripongsakun S, Wei SH, Lin S, et al. Evaluation of alpha-fetoprotein in detecting hepatocellular carcinoma recurrence after radiofrequency ablation. J Gastroenterol Hepatol. 2014;29 (1):157-164. doi:10.1111/jgh.12438

9. Zhang XF, Qi X, Meng B, et al. Prognosis evaluation in alpha-fetoprotein negative hepatocellular carcinoma after hepatectomy: comparison of five staging systems. Eur $j$ Surg Oncol. 2010;36(8):718-724. doi:10.1016/j.ejso.2010.05.022

10. Ang SF, Ng ES, Li H, et al. The Singapore Liver Cancer Recurrence (SLICER) Score for relapse prediction in patients with surgically resected hepatocellular carcinoma. PLoS One. 2015;10(4):e0118658.

11. Chan AWH, Zhong J, Berhane S, et al. Development of pre and post-operative models to predict early recurrence of hepatocellular carcinoma after surgical resection. J Hepatol. 2018;69(6):1284-1293. doi:10.1016/j.jhep.2018.08.027

12. Johnson PJ, Berhane S, Kagebayashi C, et al. Assessment of liver function in patients with hepatocellular carcinoma: a new evidence-based approach-the ALBI grade. J Clin Oncol. 2015;33 (6):550-558. doi:10.1200/JCO.2014.57.9151 
13. Yang YM, Kim SY, Seki E. Inflammation and liver cancer: molecular mechanisms and therapeutic targets. Semin Liver Dis. 2019;39 (1):26-42. doi:10.1055/s-0038-1676806

14. Xu L, Yu S, Zhuang L, et al. Systemic inflammation response index (SIRI) predicts prognosis in hepatocellular carcinoma patients. Oncotarget. 2017;8(21):34954-34960. doi:10.18632/oncotarget.16 865

15. Hu B, Yang XR, Xu Y, et al. Systemic immune-inflammation index predicts prognosis of patients after curative resection for hepatocellular carcinoma. Clin Cancer Res. 2014;20(23):6212-6222. doi:10.1158/1078-0432.CCR-14-0442

16. Wang D, Bai N, Hu X, et al. Preoperative inflammatory markers of NLR and PLR as indicators of poor prognosis in resectable HCC. PeerJ. 2019;7:e7132. doi:10.7717/peerj.7132

17. Cho CS, Gonen M, Shia J, et al. A novel prognostic nomogram is more accurate than conventional staging systems for predicting survival after resection of hepatocellular carcinoma. J Am Coll Surg. 2008;206(2):281-291. doi:10.1016/j.jamcollsurg.2007.07.031

18. Iasonos A, Schrag D, Raj GV, Panageas KS. How to build and interpret a nomogram for cancer prognosis. J Clin Oncol. 2008;26 (8):1364-1370. doi:10.1200/JCO.2007.12.9791

19. Department of Medical Administration NHaHCotPsRoC. [Guidelines for diagnosis and treatment of primary liver cancer in China (2019 edition)]. Zhonghua Gan Zang Bing Za Zhi. 2020;2(28):112-128. Chinese

20. Camp RL, Dolled-Filhart M, Rimm DL. X-tile: a new bio-informatics tool for biomarker assessment and outcome-based cut-point optimization. Clin Cancer Res. 2004;10(21):7252-7259. doi:10.1158/1078-0432.CCR-04-0713

21. Chan AW, Kumada $T$, Toyoda $H$, et al. Integration of albumin-bilirubin (ALBI) score into Barcelona Clinic Liver Cancer (BCLC) system for hepatocellular carcinoma. $J$ Gastroenterol Hepatol. 2016;31(7):1300-1306. doi:10.1111/jgh.13291

22. Hiraoka A, Kumada T, Kudo M, et al. Albumin-Bilirubin (ALBI) grade as part of the evidence-based clinical practice guideline for HCC of the Japan Society of Hepatology: a comparison with the liver damage and child-pugh classifications. Liver Cancer. 2017;6 (3):204-215. doi:10.1159/000452846

23. Wang YY, Zhong JH, Su ZY, et al. Albumin-bilirubin versus Child-Pugh score as a predictor of outcome after liver resection for hepatocellular carcinoma. $B r \quad J \quad$ Surg. 2016;103(6):725-734. doi:10.1002/bjs. 10095

24. Li MX, Zhao H, Bi XY, et al. Prognostic value of the albumin-bilirubin grade in patients with hepatocellular carcinoma: validation in a Chinese cohort. Hepatol Res. 2017;47(8):731-741. doi:10.1111/hepr.12796

25. Ho SY, Hsu CY, Liu PH, et al. Albumin-bilirubin (ALBI) grade-based nomogram to predict tumor recurrence in patients with hepatocellular carcinoma. Eur j Surg Oncol. 2019;45(5):776-781. doi:10.1016/j.ejso.2018.10.541

26. He W, Peng B, Tang Y, et al. Nomogram to predict survival of patients with recurrence of hepatocellular carcinoma after surgery. Clin Gastroenterol Hepatol. 2018;16(5):756-764 e710. doi:10.1016/ j.cgh.2017.12.002

27. Llovet JM, Zucman-Rossi J, Pikarsky E, et al. Hepatocellular carcinoma. Nat Rev Dis Primers. 2016;2:16018.

28. Ramakrishna G, Rastogi A, Trehanpati N, Sen B, Khosla R, Sarin SK. From cirrhosis to hepatocellular carcinoma: new molecular insights on inflammation and cellular senescence. Liver Cancer. 2013;2(3-4):367-383. doi:10.1159/000343852
29. Chen Q, Li F, Zhong C, et al. Inflammation score system using preoperative inflammatory markers to predict prognosis for hepatocellular carcinoma after hepatectomy: a cohort study. J Cancer. 2020;11(17):4947-4956. doi:10.7150/jca.45274

30. Lippitz BE, Harris RA. Cytokine patterns in cancer patients: a review of the correlation between interleukin 6 and prognosis. Oncoimmunology. 2016;5(5):e1093722. doi:10.1080/2162402X.2015.1093722

31. Grivennikov SI, Greten FR, Karin M. Immunity, inflammation, and cancer. Cell. 2010;140(6):883-899. doi:10.1016/j.cell.2010.01.025

32. Song W, Tian C, Wang K, Zhang RJ, Zou SB. The pretreatment lymphocyte to monocyte ratio predicts clinical outcome for patients with hepatocellular carcinoma: a meta-analysis. Sci Rep. 2017;7:46601. doi:10.1038/srep46601

33. Wu Y, Tu C, Shao C. Inflammatory indexes in preoperative blood routine to predict early recurrence of hepatocellular carcinoma after curative hepatectomy. BMC Surg. 2021;21(1):178. doi:10.1186/ s12893-021-01180-9

34. Roayaie S, Blume IN, Thung SN, et al. A system of classifying microvascular invasion to predict outcome after resection in patients with hepatocellular carcinoma. Gastroenterology. 2009;137 (3):850-855. doi:10.1053/j.gastro.2009.06.003

35. Rodríguez-Perálvarez M, Luong TV, Andreana L, Meyer T, Dhillon AP, Burroughs AK. A systematic review of microvascular invasion in hepatocellular carcinoma: diagnostic and prognostic variability. Ann Surg Oncol. 2013;20(1):325-339. doi:10.1245/ s10434-012-2513-1

36. Wang L, Jin YX, Ji YZ, Mu Y, Zhang SC, Pan SY. Development and validation of a prediction model for microvascular invasion in hepatocellular carcinoma. World j Gastroenterol. 2020;26(14):1647-1659. doi:10.3748/wjg.v26.i14.1647

37. Li X, Huang H, Yu X, Chen P, Ouyang J, Huang B. A novel prognostic nomogram based on microvascular invasion and hematological biomarkers to predict survival outcome for hepatocellular carcinoma patients. Surg Oncol. 2020;33:51-57. doi:10.1016/j.suronc.2020.01.006

38. Lee S, Kang TW, Song KD, et al. Effect of Microvascular invasion risk on early recurrence of hepatocellular carcinoma after surgery and radiofrequency ablation. Ann Surg. 2021;273(3):564-571. doi:10.1097/SLA.0000000000003268

39. Huang J, Liu FC, Li L, Zhou WP, Jiang BG, Pan ZY. Nomograms to predict the long-time prognosis in patients with alpha-fetoprotein negative hepatocellular carcinoma following radical resection. Cancer Med. 2020;9(8):2791-2802. doi:10.1002/cam4.2944

40. Senzolo M, Burra P, Cholongitas E, Burroughs AK. New insights into the coagulopathy of liver disease and liver transplantation. World j Gastroenterol. 2006;12(48):7725-7736. doi:10.3748/wjg.v12.i48.7725

41. Wang Y, Sun K, Shen J, et al. Novel prognostic nomograms based on inflammation-related markers for patients with hepatocellular carcinoma underwent hepatectomy. Cancer Res Treatment. 2019;51 (4):1464-1478. doi:10.4143/crt.2018.657

42. Haruki K, Shiba H, Saito N, et al. Risk stratification using a novel liver functional reserve score of combination prothrombin time-international normalized ratio to albumin ratio and albumin in patients with hepatocellular carcinoma. Surgery. 2018;164 (3):404-410. doi:10.1016/j.surg.2018.02.022

43. Pajouheshnia R, Pestman WR, Teerenstra S, Groenwold RH. A computational approach to compare regression modelling strategies in prediction research. BMC Med Res Methodol. 2016;16(1):107. doi:10.1186/s12874-016-0209-0

44. Moons KG, Kengne AP, Woodward M, et al. Risk prediction models: i. Development, internal validation, and assessing the incremental value of a new (bio)marker. Heart. 2012;98(9):683-690. 


\section{Publish your work in this journal}

The Journal of Hepatocellular Carcinoma is an international, peerreviewed, open access journal that offers a platform for the dissemination and study of clinical, translational and basic research findings in this rapidly developing field. Development in areas including, but not limited to, epidemiology, vaccination, hepatitis therapy, pathology and molecular tumor classification and prognostication are all considered for publication. The manuscript management system is completely online and includes a very quick and fair peer-review system, which is all easy to use. Visit http://www.dovepress.com/ testimonials.php to read real quotes from published authors.

Submit your manuscript here: https://www.dovepress.com/journal-of-hepatocellular-carcinoma-journal 